\title{
Dielectric Properties Tangential to the Interface in Model Insoluble Monolayers - Theoretical Assessment
}

\author{
(Supporting Information)
}

Ph. Shushkov, S. Tzvetanov, A. Ivanova, A. Tadjer*

University of Sofia, Faculty of Chemistry, 1 James Bourchier blvd., 1164 Sofia, Bulgaria

Table S1: Parameters of the MC and MD simulations for each elementary cell (EC): area per surfactant $(A)$ and dimensions of the periodic box.

\begin{tabular}{|c|c|c|}
\hline $\begin{array}{c}\text { Surfactants } \\
\text { in EC }\end{array}$ & $\boldsymbol{A}^{\AA^{2}}$ & Box size, $\AA$ \\
\hline \multirow{4}{*}{ 9 DPPC } & 40 & $19.0 \times 19.0 \times 100.0$ \\
\cline { 2 - 3 } & 50 & $21.2 \times 21.2 \times 100.0$ \\
\cline { 2 - 3 } & 60 & $23.3 \times 23.3 \times 100.0$ \\
\cline { 2 - 3 } & 70 & $25.1 \times 25.1 \times 100.0$ \\
\cline { 2 - 3 } & 80 & $26.8 \times 26.8 \times 100.0$ \\
\cline { 2 - 3 } & 100 & $30.0 \times 30.0 \times 100.0$ \\
\hline \hline \multirow{4}{*}{ 9 DC } & 120 & $32.9 \times 32.9 \times 100.0$ \\
\cline { 2 - 3 } & 40 & $13.5 \times 27.0 \times 100.0$ \\
\cline { 2 - 3 } & 50 & $15.0 \times 30.0 \times 100.0$ \\
\cline { 2 - 3 } & 60 & $17.4 \times 30.9 \times 100.0$ \\
\cline { 2 - 3 } & 70 & $17.8 \times 35.5 \times 100.0$ \\
\cline { 2 - 3 } & 100 & $21.0 \times 34.5 \times 100.0$ \\
\cline { 2 - 3 } & 120 & $26.9 \times 40.4 \times 100.0$ \\
\hline
\end{tabular}



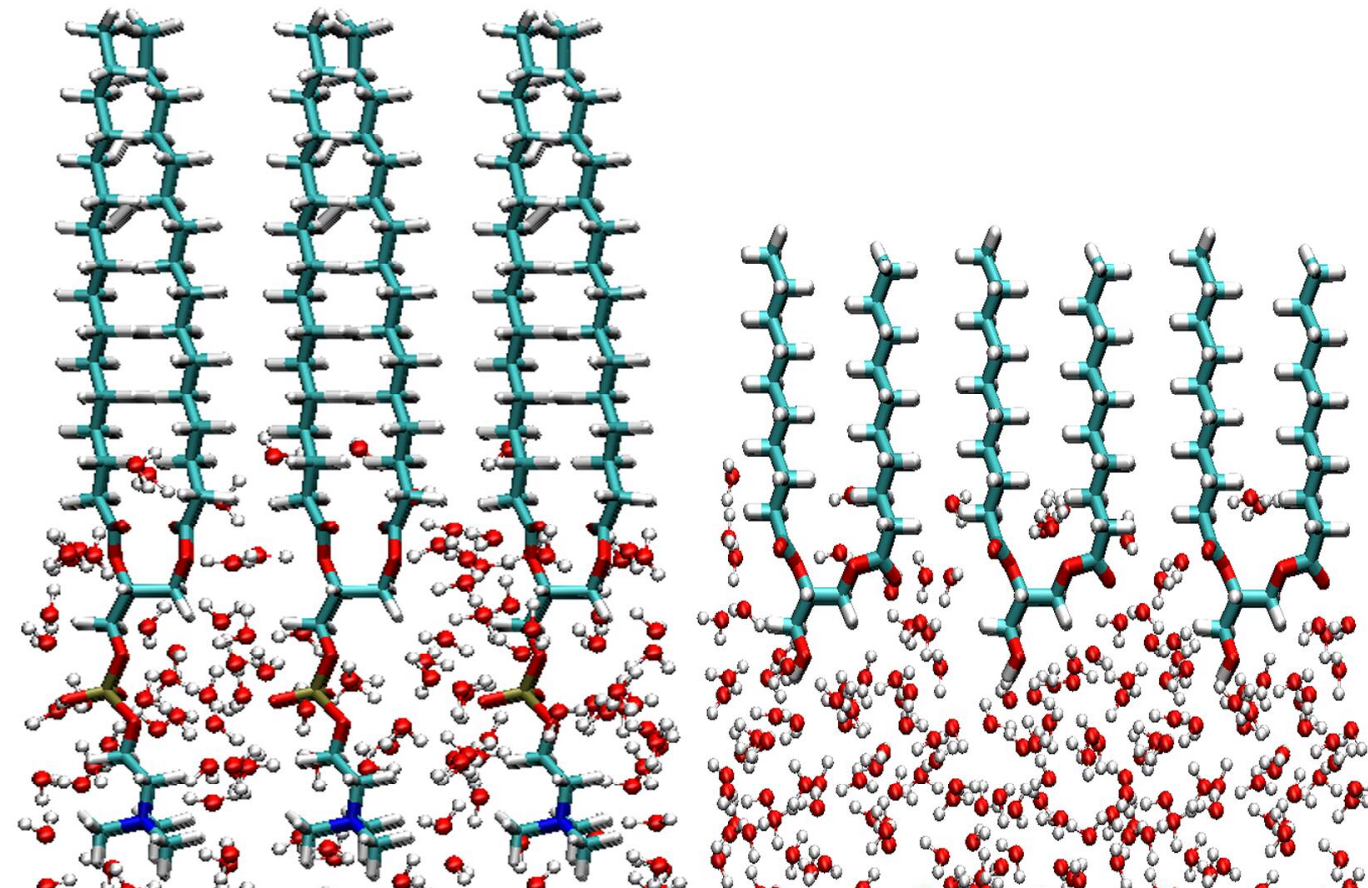

800 d o o o ar ze are

s 900 o

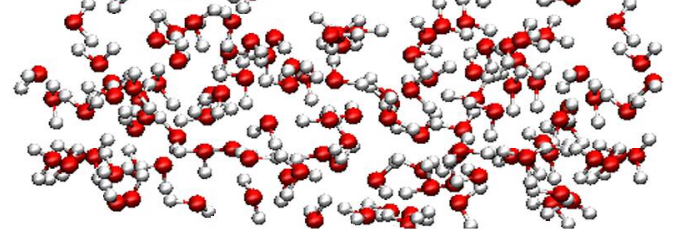

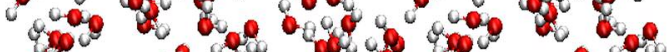

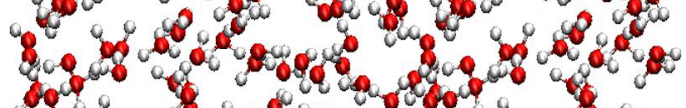

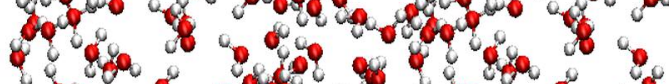

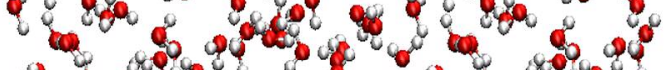

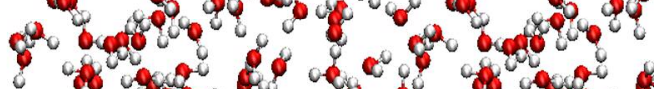

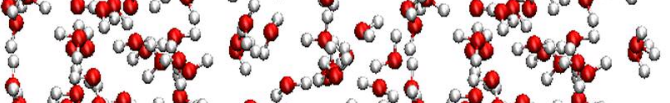

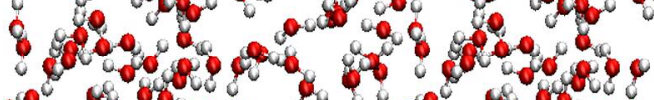

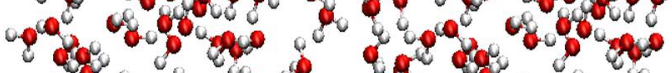

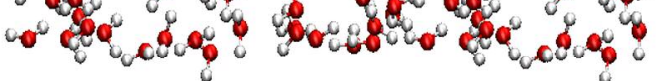
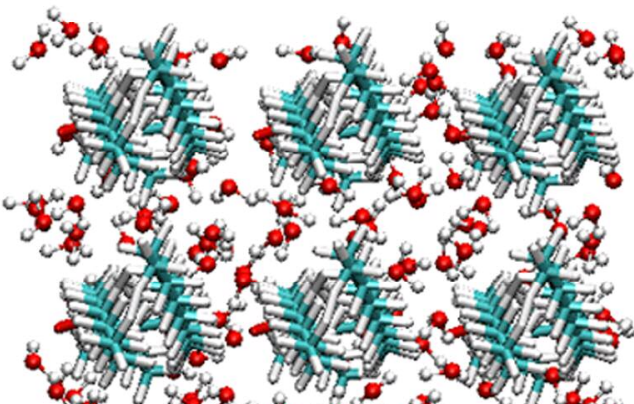

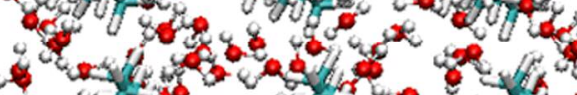
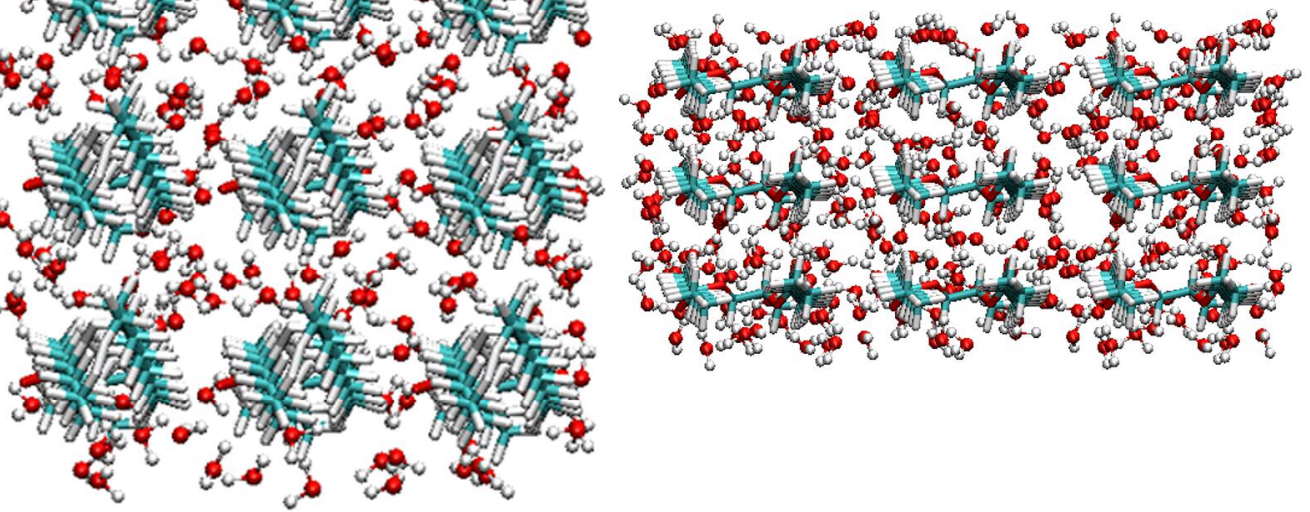

Figure S1: Initial elementary cells at $50 \AA^{2} /$ molec. for DPPC (left) and DC (right) simulations: side view (top), top view (bottom). 


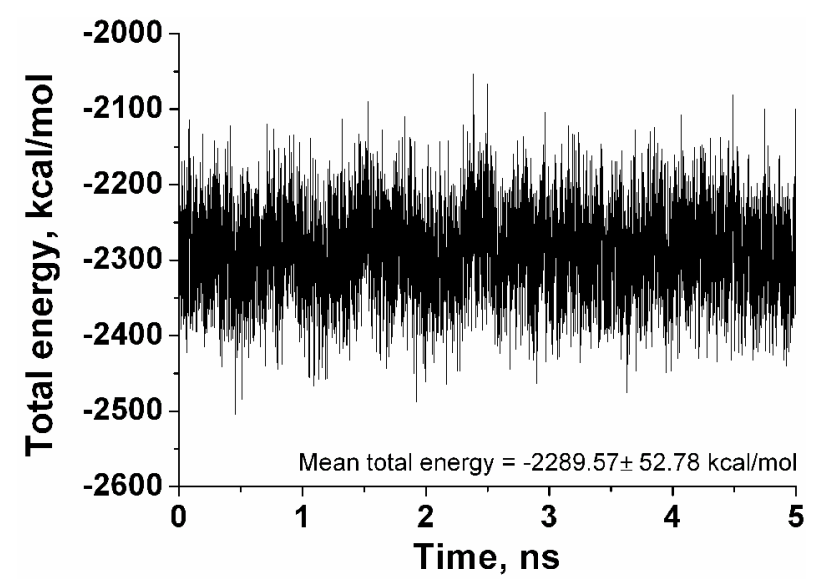

Figure S2: Total energy profile of the production MD simulation of DPPC monolayer at $120 \AA^{2} /$ molecule. 

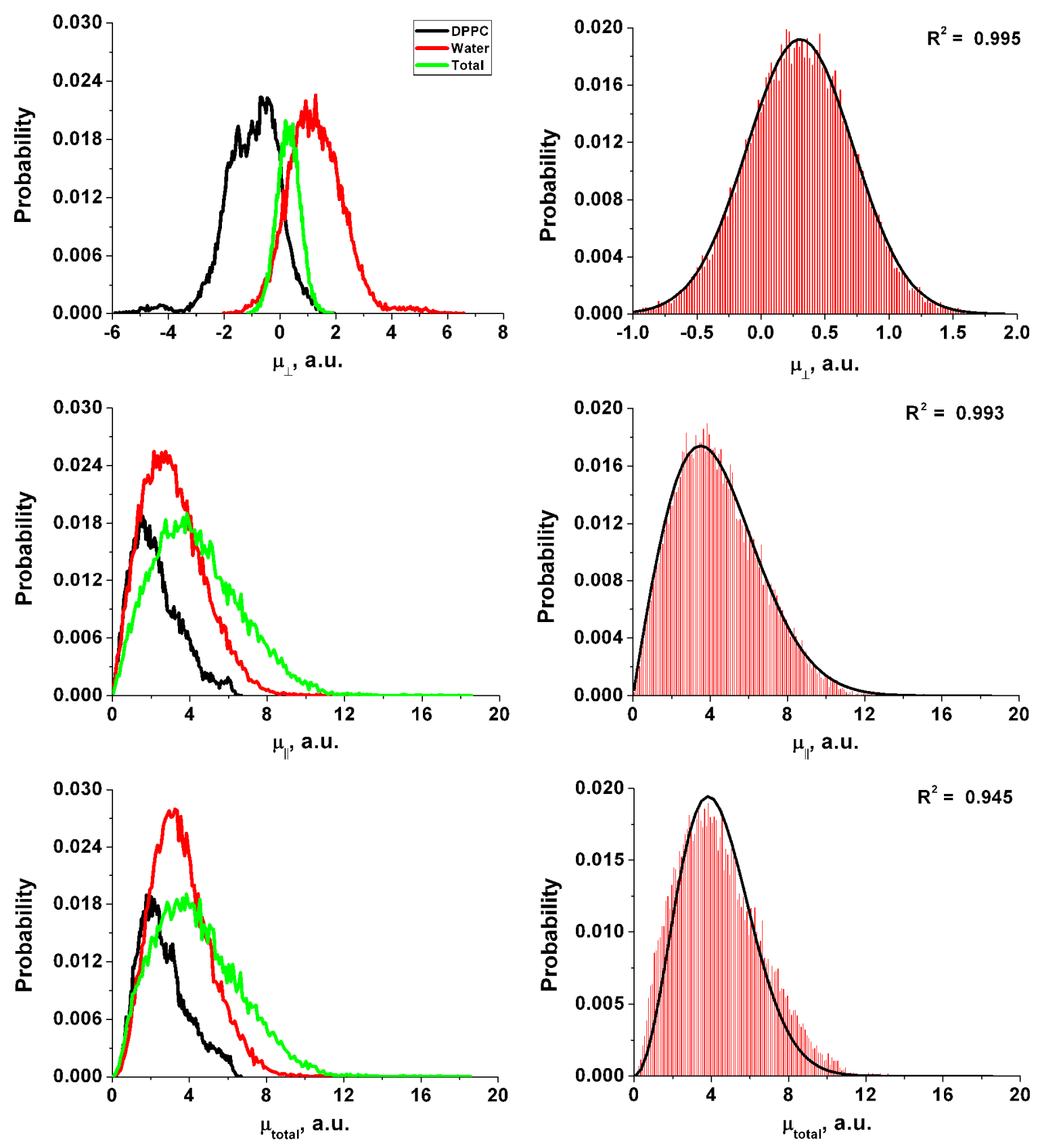

Figure S3: Illustrative probability distributions of the calculated dipole moments (DPPC monolayer at 120 $\AA^{2} /$ molecule). Contributions from water and surfactant (left) as well as bin distribution of the total dipole moment and its components $\left(\mu_{\perp}\right.$ and $\left.\mu_{\|}\right)$with corresponding Gaussian or Boltzmann fits (right) are shown. 

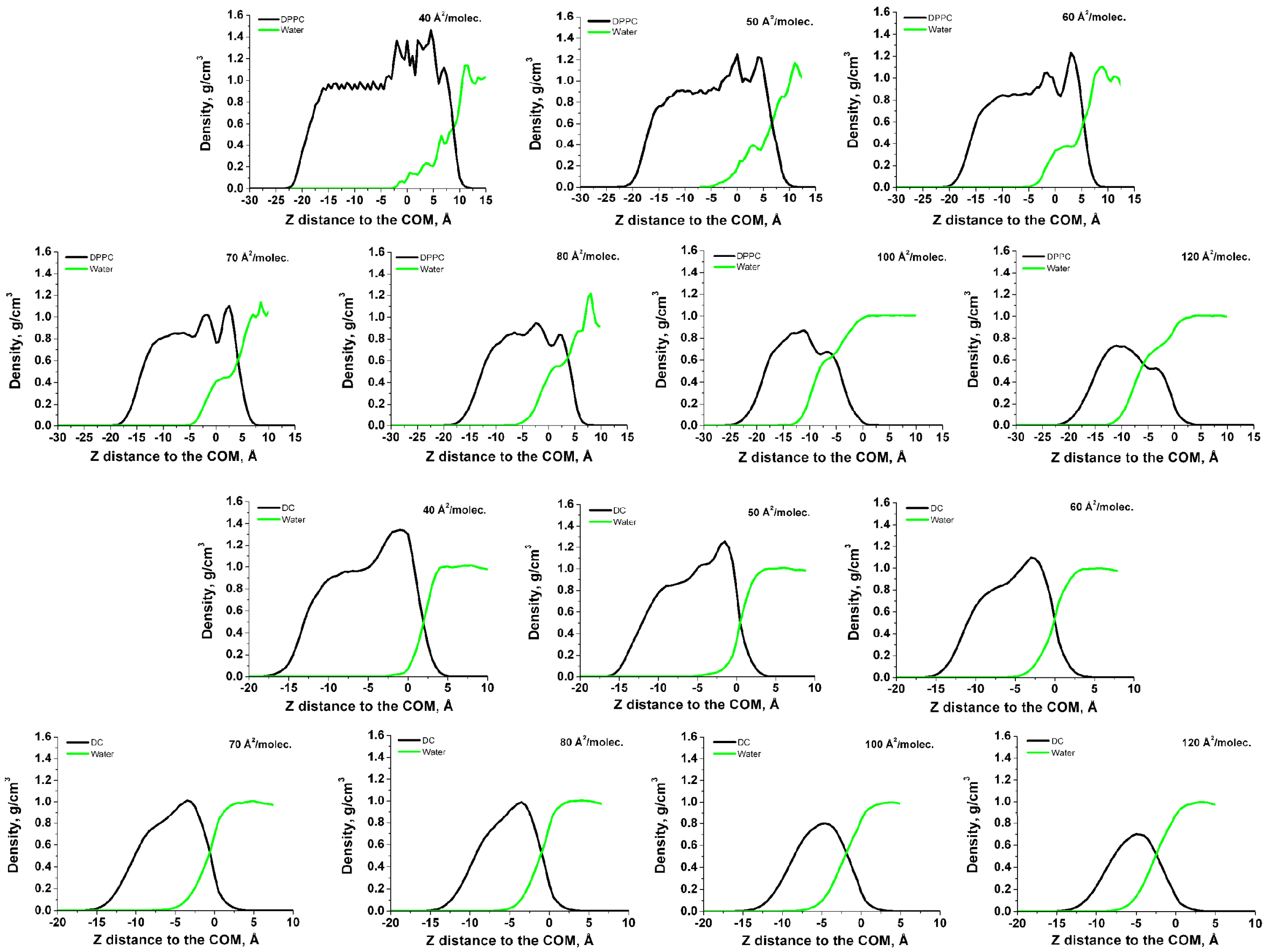

Figure S4: Density profiles of DPPC and DC monolayers and contributions from surfactant and water in direction normal to the interface 

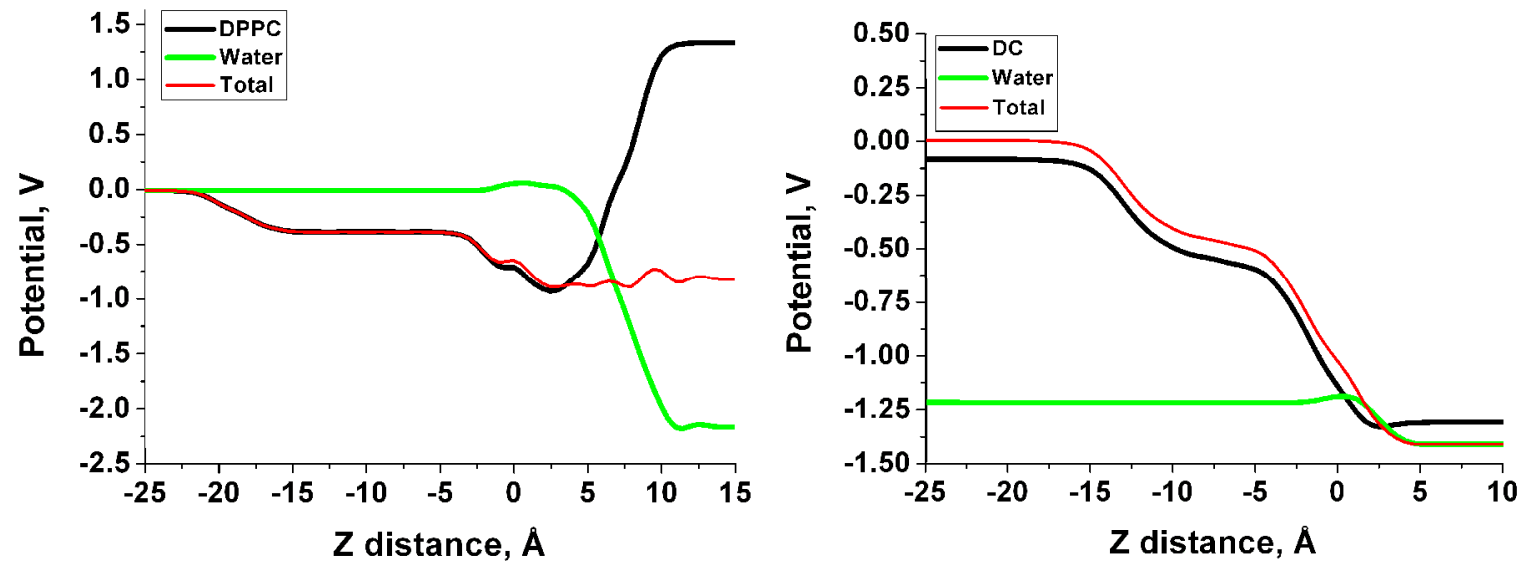

Figure S5: Illustration of the potential profile across DPPC (left, $70 \AA^{2} /$ molecule) and DC (right, 40 $\AA^{2} /$ molecule) monolayers and the contributions of water (green) and surfactant (black) to the total values (red). 